\title{
Estimulación nerviosa eléctrica transcutánea como complemento a la terapia convencional en pacientes con trastornos temporomandibulares: un estudio caso-control
}

\section{Transcutaneous electrical nerve stimulation as a complement to conventional therapy in patients with temporomandibular disorders: a case-control study}

W. Meeder Bella*, C. León-Morales*, O. Leissner*, E. Vergara-Bruna*, M. Maulén-Yañez*, W. A. González-Arriagada**

\section{RESUMEN}

Introducción: Para los trastornos temporomandibulares (TTM) el tratamiento de primera línea es el conservador, éste incluye a la terapia convencional, además se ha descrito el uso de la estimulación nerviosa eléctrica transcutánea (TENS). El objetivo de este estudio fue determinar la respuesta terapéutica del TENS como complemento a la terapia convencional comparado con pacientes que recibieron exclusivamente terapia convencional.

Material y Métodos: Estudio retrospectivo caso-control en pacientes diagnosticados con TTM, tratados en la Clínica de Especialidades de la Escuela de Odontología de la Universidad de Valparaíso, entre los años 2009 y 2012. De los 231 pacientes, 63 cumplieron con los criterios de inclusión y fueron pareados en un grupo convencional y un grupo TENS. Se evaluó el dolor articular y muscular; aperturas mandibulares activa sin dolor, activa forzada y pasiva; y fuerza masticatoria. Se aplicaron test estadísticos de Kruskal-Wallis, Wilcoxon y Spearman.

Resultados: Para el grupo TENS, se encontraron respuestas significativas en dolor articular y muscular derecho e izquierdo, apertura mandibular activa sin dolor y fuerza masticatoria. Para el grupo convencional, se encontraron resultados similares a excepción de dolor articular derecho. También respuestas significativas para todas las variables que midieron dolor inmediatamente posterior a la aplicación de TENS. Finalmente se establecieron correlaciones entre la edad y las aperturas activa sin dolor, activa forzada y pasiva.

Discusión: La terapia convencional y la complementada con TENS demostraron similar respuesta terapéutica a largo plazo, sin embargo, la aplicación del TENS produce una respuesta inmediata que reduce el dolor en el corto plazo.

PALABRAS CLAVE: Trastornos de la articulación Temporomandibular, escala visual análoga, apertura bucal, fuerza de mordida, terapia complementaria.

* Departamento de Fisiología Oral y Trastornos Temporomandibulares, Facultad de Odontología, Universidad de Valparaíso, Valparaíso, Chile.

* * Departamento de Patología Oral y Diagnóstico, Facultad de Odontología, Universidad de Valparaíso, Valparaíso, Chile.

Centro de Investigación en Ciencias Odontológicas y Médicas (CICOM), Universidad de Valparaíso, Valparaíso, Chile. 


\begin{abstract}
Introduction: For temporomandibular disorders (TMD), conservative therapies, including conventional therapy, are the first line of treatment, and the use of transcutaneous electrical nerve stimulation (TENS) has also been reported. The aim of this study was to determine the therapeutic response to TENS as complement to conventional therapy compared with patients receiving conventional therapy exclusively. Methodology: A retrospective study was carried out with patients diagnosed with TMD, treated at the Clinic of Specialties of the Dental School of Universidad de Valparaíso, between 2009 and 2012. Of 231 patients, 63 met the inclusion criteria and were randomly allocated to a conventional therapy group (controls) and a coadjuvant TENS group (cases). Both were evaluated for pain in the temporomandibular joints (TMJ) and masseters, active opening without pain, forced active opening, passive opening, and occlusal force. Statistical analysis was performed with Kruskal-Wallis, Wilcoxon and Spearman tests.

Results: For TENS group, significant differences were found for pain in the right and left TMJ, pain in the right and left masseter, active opening without pain, and occlusal force. For the conventional group, the same results were obtained, except for pain in right TMJ. For the variables measured immediately after applying TENS, significant differences were found in all pain variables. Correlations were established with age, active opening without pain, forced active opening, and passive opening.

Discussion: Conventional therapy and TENS-complemented therapy showed good therapeutic response, concluding that TENS is a good complement to conventional therapy.
\end{abstract}

KEY WORDS: TMD; visual analog scale; mouth opening; masticatory force; complementary therapy.

Fecha de recepción: 25 de marzo de 2020

Fecha de aceptación: 25 de marzo de 2020

W. Meeder Bella, C. León-Morales, O. Leissner, E. Vergara-Bruna, M. Maulén-Yañez, W. A. González-Arriagada. Estimulación nerviosa eléctrica transcutánea como complemento a la terapia convencional en pacientes con trastornos temporomandibulares: un estudio caso-control. 2020; 36, (4): 208-217.

\section{INTRODUCCIÓN}

Los trastornos temporomandibulares (TTM) son un grupo de condiciones musculoesqueletales y neuromusculares que involucran a las articulaciones temporomandibulares (ATM), los músculos masticadores, y los tejidos asociados ${ }^{(1)}$. La prevalencia de los TTM se ha descrito entre un 5 a un $12 \%$ en la población en general, siendo más prevalente en mujeres entre los 25 y 45 años de edad ${ }^{(2)}$.

Su etiología y patogénesis se explican mediante una secuencia en la que la presencia de eventos locales o sistémicos puede ultrapasar un umbral de tolerancia fisiológica, induciendo el inicio de los síntomas ${ }^{(3)}$. Es por ello, que actualmente se les atribuye una etiología multifactorial, evidenciada por una combinación de factores psicológicos, fisiológicos, neuromusculares, parafuncionales, traumáticos, estructurales, hormonales, posturales y genéticos, los cuales alteran el ba- lance funcional de los elementos fundamentales del sistema estomatognático: oclusión, músculos y $\operatorname{ATM}^{(4)}$.

La clasificación diagnóstica de los TTM puede basarse en los criterios de la Asociación Americana de Dolor Orofacial(AAOP) ${ }^{(1)}$ o en los Criterios Diagnósticos para los Trastornos Temporomandibulares (DC/TMD) ${ }^{(2)}$. El diagnóstico de los TTM se basa en la historia y el examen clínico del paciente ${ }^{(5)}$, auxiliado por herramientas como la electromiografía (EMG), la cual identifica las características de los músculos masticadores durante su funcionamiento en tareas dinámicas o estáticas en pacientes con TTM, lo cual puede ser comparado con pacientes sanos. Este enfoque puede ser usado para evaluar la eficacia de diferentes terapias ${ }^{(6)}$.

Los objetivos del tratamiento de los TTM son la reducción y/o eliminación del dolor, y la recuperación de la correcta función del sistema estoma- 
tognático ${ }^{(3)}$. El tratamiento es complejo, requiere de un adecuado diagnóstico y de conocimientos específicos por parte del paciente y del profesional.

Estas terapias pueden ser clasificadas como conservadoras, mínimamente invasivas o invasivas $^{(7)}$. Dentro de las terapias conservadoras encontramos a la terapia convencional (TC), la cual involucra el uso de terapia ortopédica (TO) y la auto-regulación física (ARF) ${ }^{(8)}$. Otras terapias conservadoras incluyen a la estimulación nerviosa eléctrica transcutánea o TENS, por sus siglas en inglés, la cual ha sido usada para controlar el dolor en pacientes con TTM ${ }^{(7)}$, logrando una mejora en el manejo del dolor y disfunción. Sin embargo los distintos parámetros usados para evaluar su eficacia, tanto sintomatológicos como funcionales, han arrojado resultados no concluyentes en pacientes con TTM tanto en el corto como largo plazo ${ }^{(9)}$.

\section{ESTIMULACIÓN NERVIOSA ELÉCTRICA TRANSCUTÁNEA (TENS)}

El TENS es un dispositivo que emite pulsos eléctricos de baja o alta frecuencia y de baja o alta intensidad, en la superficie de la piel, trasmitidos por electrodos posicionados cerca o en el sitio del dolor, donde el paciente percibe una sensación de burbujeo o contracciones fibrilares. Se ha descrito como un método seguro, de bajo costo y con opción de ser auto medicado, logrando analgesia inducida por neuroestimulación, alivio o bloqueo sin efectos adversos. Este perfil de seguridad lo compara positivamente ante otras terapias ${ }^{(10)}$.

Los mecanismos que utilizan se explican mediante dos teorías, la teoría de la compuerta y la de opioides endógenos; a las cuales se les ha agregado un tercer componente que es el efecto placebo ${ }^{(11)}$.

Además se ha reportado que el tiempo de aplicación por sesión de TENS debe ser mínimo aproximadamente 30-40 minutos para lograr relajación muscular comprobada por EMG tanto en individuos enfermos como sanos ${ }^{(12)}$.

Para el manejo de los TTM estudios reportaron que el uso de TENS logra una disminución del dolor y actividad electromiográfica de maséteros ${ }^{(13)}$, en la misma línea se ha reportado que el TENS reduce el dolor muscular en pacientes con TTM de tipo muscular ${ }^{(14)}$, y en otro aspecto se reportó que el TENS contribuye al aumento del rango de apertura mandibular en pacientes con TTM ${ }^{(15)}$. Por otro lado para el manejo de dolor crónico en pacientes con TTM el uso del TENS es controversial ${ }^{(16)}$, pero su importancia en el manejo del dolor de músculos masticatorios es significativa ${ }^{(17 ; 18)}$ y puede ser usado como complemento en el manejo del dolor asociado a TTM ${ }^{(19 ; 20)}$. Adicionalmente recientemente se reportó que el uso de TENS en pacientes con TTM refractarios a tratamiento farmacológico con resultados satisfactorios ${ }^{(21)}$.

Considerando los reportes inconclusos en relación a la utilidad del TENS en los TTM, el objetivo de este estudio fue determinar la respuesta terapéutica al TENS como complemento a la terapia convencional en el tratamiento de pacientes con TTM comparado con pacientes que reciben terapia convencional exclusiva.

\section{MATERIALES Y MÉTODOS}

\section{Diseño Y RECOLECCIÓN DE DATOS}

El presente fue un estudio retrospectivo caso-control que se desarrolló mediante una base de datos anonimizada en la cual se censaron todas las fichas clínicas de entre mayo del año 2009 y octubre de 2012, correspondientes a pacientes que consultaron directamente o fueron derivados a la Clínica de Especialidades de la Facultad de Odontología de la Universidad de Valparaíso. El universo de población estudiada fueron 231 pacientes diagnosticados con TTM a los que luego se les aplicaron criterios de inclusión y exclusión obteniendo una muestra total de 63 pacientes. La muestra fue dividida en dos grupos de pacientes, uno con TTM que recibieron terapia convencional complementada con TENS $(n=32)$ y un segundo grupo con TTM que recibieron terapia convencional exclusivamente $(n=31)$, con un control por cada caso. Los pacientes fueron pareados por rangos de edad (bajo y sobre 25 años) y por rango de dolor muscular y articular (bajo y sobre 5 en escala visual análoga). Todos los pacientes firmaron un consentimiento informado escrito previo al tratamiento. Este estudio fue aprobado por el comité de bioética de la Facultad de Odontología de la Universidad de Valparaíso, código de aprobación PostG-08-17. No existieron conflictos de interés por parte de ninguno de los investigadores.

Los criterios de inclusión fueron ser mayores de 
14 años, la presencia completa de dientes anteriores, poseer menos de 5 piezas ausentes posteriores, anamnesis positiva en al menos una pregunta del cuestionario de la AAOP (1), dolor mayor o igual a EVA 2 en al menos un masétero o ATM.

Los criterios de exclusión incluyeron embarazadas; portadores de marcapasos; enfermedades sistémicas asociadas (poliartropatías, diabetes, otros trastornos músculo esqueletales); enfermedades psiquiátricas diagnosticadas; en tratamiento con fármacos antidepresivos, ansiolíticos o hipnóticos; y diagnóstico clínico de luxación disco-condilar con reducción. Los criterios diagnósticos utilizados se basan en las pautas de investigación RCD/TMD de Dworkin ${ }^{(22)}$.

\section{TERAPIA}

Para el grupo control (CONV) se utilizó TC consistente en el uso de TO con uso nocturno de plano de Michigan, plano de cobertura total, el cual se ajustó en controles posteriores a su instalación. Además se realizó $A R F$ la que consistió en la reducción de parafunciones musculares, entrenamiento propioceptivo para mejorar la postura de cuello, cabeza y mandíbula, higiene de sueño, actividad física, eliminación de malos hábitos y la corrección de la posición postural mandibular, asesoramiento nutricional y ejercicios de respiración para relajación.

Para los casos (grupo TENS), además de lo anteriormente mencionado se aplicó en cada sesión de tratamiento TENS por 30 minutos mediante electrodos posicionados en la piel en el sitio del dolor. El dispositivo usado fue TS-1211 TENS (CDM Medical, Las Condes, Santiago, Chile), y el modo usado programado varió la frecuencia de pulso y su ancho de banda automáticamente para evitar que los pacientes generaran tolerancia analgésica al TENS. La intensidad fue ajustada de acuerdo a la sensibilidad de cada paciente ${ }^{(23)}$. Los clínicos fueron 3 académicos especialistas en TTM, los cuales se calibraron en examen físico, uso de TENS, pie de metro y gnatodinamómetro, esto como parte del entrenamiento de rutina del programa de postgrado. El índice Cohen Kappa final fue de 0.92 .

Las características socio demográficas independientes y el tipo de terapia administrada fueron recuperadas de los archivos de ingreso. Las variables dependientes fueron medidas sesión a sesión, con una medición extra para variables que midie-

\begin{tabular}{|c|c|}
\hline Edad & Registrada en años. \\
\hline Género & Registrado como masculino o femenino. \\
\hline TENS complemento a TC & Asignada al momento de iniciar el tratamiento. \\
\hline Dolor articular & $\begin{array}{l}\text { Dolor evaluado mediante EVA (1 a 10). Se registra mediante la } \\
\text { palpación del polo externo de la ATM (presión de } 0.5 \mathrm{~kg} \text { por } 2 \\
\text { seg) pudiendo haber dolor localizado o difuso. }\end{array}$ \\
\hline Dolor maseterino & $\begin{array}{l}\text { Dolor evaluado mediante la EVA (1 a 10). Se registra pal- } \\
\text { pando el músculo masétero (presión de } 1 \mathrm{~kg} \text { por } 2 \text { seg) } \\
\text { pudiendo haber dolor localizado, irradiado o referido. }\end{array}$ \\
\hline Apertura activa sin dolor & $\begin{array}{l}\text { Apertura máxima en milímetros del paciente en la cual no } \\
\text { hay dolor. Se mide con el pie de metro, la separación entre los } \\
\text { bordes incisales de los incisivos centrales superiores e inferiores } \\
\text { derechos (FDI 1.1 y 4.1) en el plano frontal. }\end{array}$ \\
\hline Apertura activa forzada & $\begin{array}{l}\text { Apertura máxima en milímetros por parte del paciente en la cual } \\
\text { puede haber dolor. Se mide con el pie de metro, la separación } \\
\text { entre los bordes incisales de los incisivos centrales superiores e } \\
\text { inferiores derechos (FDI 1.1 y 4.1) en el plano frontal. }\end{array}$ \\
\hline Apertura pasiva & $\begin{array}{l}\text { Apertura máxima en milímetros con ayuda del operador en la cual } \\
\text { puede haber dolor, se realiza presionando con el dedo índice y } \\
\text { pulgar ( } 1 \mathrm{~kg} \text { por } 2 \text { seg). Se mide con el pie de metro, la separación } \\
\text { entre los bordes incisales de los incisivos centrales superiores e } \\
\text { inferiores derechos (FDl } 1.1 \text { y } 4.1 \text { ) en el plano frontal. }\end{array}$ \\
\hline Fuerza masticatoria & $\begin{array}{l}\text { Registrada gracias a un gnatodinamómetro, instrumento el cual } \\
\text { se utiliza en los caninos derechos (FDI 1.3 y 4.3), y mide en kiló- } \\
\text { gramos la fuerza masticatoria máxima ejercida por el paciente. }\end{array}$ \\
\hline
\end{tabular}

$\mathbf{A T M}=$ Articulación Temporomandibular, $\mathbf{T C}=$ Terapia convencional, EVA= Escala visual análoga, $\mathrm{FDI}=$ Federación dental Internacional.

\begin{tabular}{|c|c|c|c|}
\hline Sesión 1: & Evaluación y toma de impresión & Registro de Variables & Tto. con ARF y TENS \\
\hline Sesión 2: & Registro de cera & Registro de Variables & Tto. con ARF y TENS \\
\hline Sesión 3: & Instalación & Registro de variables & Tto. con ARF y TENS \\
\hline Sesiones 4-8: & Ajuste plano & Registro de variables & Tto. con ARF y TENS \\
\hline Sesiones 9-11: & Control de plano & Registro de variables & Tto. con ARF y TENS \\
\hline
\end{tabular}

Tto $=$ Tratamiento, $\mathbf{A R F}=$ Auto regulación física, $\mathbf{T E N S}=$ Transcutaneous electrical nerve stimulation.

ron dolor en el grupo de estudio inmediatamente posterior a la aplicación de TENS (Tabla 1).

Luego de la evaluación, se consideraron 8 sesiones de tratamiento (Tabla 2), con una distribución de 2 sesiones semanales por mes. Además de 3 sesiones de control, a 15 días, 30 días y finalmente 60 días luego del término del tratamiento, con un máximo de 11 sesiones en total. Sin embargo, esta sistemática no fue mantenida por todos los pacientes del estudio.

\section{AnÁlisis estadístico}

El análisis estadístico se realizó mediante el software estadístico SPSS IBM Statistics versión 21.0. Para verificar la normalidad de la distribución se utilizó test de Shapiro-Wilk. Luego para las variables independientes y los valores iniciales de las dependientes se utilizó test de Chi Cuadrado de Pearson. Posteriormente para evaluar la res- 
puesta terapéutica para todas las variables medidas entre los valores iniciales y finales, dentro de cada grupo, se aplicó test de Kruskal-Wallis. A continuación para medir el efecto inmediato de la aplicación del TENS en las variables que midieron dolor articular y muscular se utilizó test de Wilcoxon. Finalmente para establecer correlaciones primero entre el número de sesiones y luego entre la edad de los pacientes y cada una de las variables se utilizó test de Correlación de Spearman. Aplicando un valor $\mathrm{p}<0.05$ y un $\mathrm{IC}=95 \%$.

\section{RESULTADOS}

En este estudio, la totalidad de los pacientes ( $n=63)$, fue dividida en dos grupos, uno tratado con TENS como complemento (TENS) con 32 pacientes (50.8\%), y otro con terapia convencional (CONV) con 31 pacientes (49.2\%). Durante el seguimiento, 5 pacientes abandonaron el estudio, por lo que solo 28 pacientes del grupo TENS y 30 del grupo CONV fueron analizados. La selección de los pacientes y su inclusión son descritas en el siguiente flujograma (Figura 1):

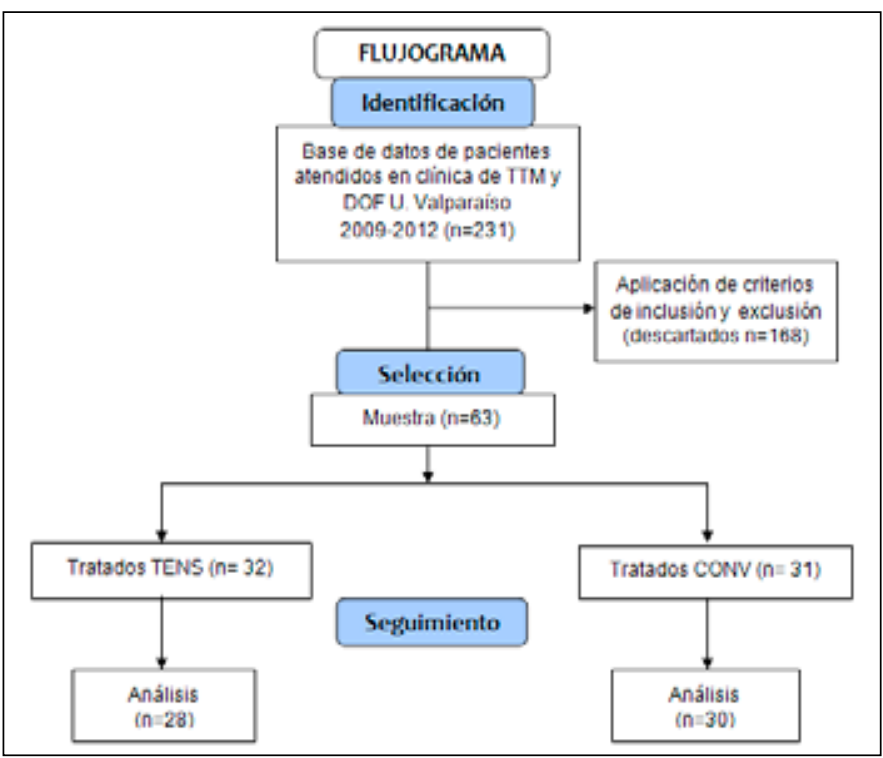

Figura 1. Flujograma con identificación, selección y seguimiento de los pacientes.

Los pacientes presentaron un rango de edad entre 14 y 69 años, con un promedio de edad de 29 años $( \pm 11.1)$. Del total de pacientes, $8(12.7 \%)$ correspondieron a sexo masculino y 55 (87.3\%) a femenino. El porcentaje y distribución de los pacientes según edad y sexo en los grupos CONV y TENS se describe a continuación (Tabla 3).

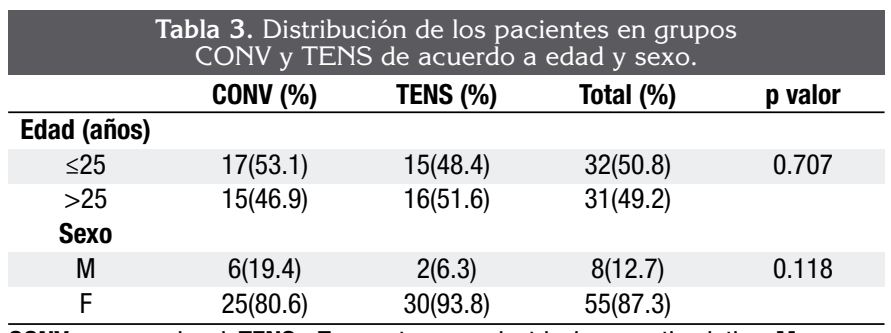

$\mathbf{C O N V}=$ convencional, TENS $=$ Transcutaneous electrical nerve stimulation, $\mathbf{M}=$ masculino, $\mathbf{F}=$ femenino.

\begin{tabular}{|c|c|c|c|c|c|}
\hline & $\begin{array}{l}\text { 4. Dis } \\
\text { is varia }\end{array}$ & $\begin{array}{l}\text { ución } \\
\text { s en } g\end{array}$ & $\begin{array}{l}\text { os valor } \\
\text { s CON }\end{array}$ & $\begin{array}{l}\text { es iniciales } \\
\text { V y TENS. }\end{array}$ & \\
\hline & CONV(\%) & TENS(\%) & Total (\%) & promedio ( $(\mathrm{SD})$ & p valor \\
\hline EVA ATM der in & & & & & \\
\hline$\leq 5$ & $22(44)$ & $28(56)$ & $50(79.4)$ & $2.7( \pm 2.662)$ & $p=0.105$ \\
\hline$>5$ & $9(69.2)$ & $4(30.8)$ & 13(20.6) & & \\
\hline EVA ATM izq ini & & & & & \\
\hline$\leq 5$ & $23(50)$ & $23(50)$ & $46(73)$ & $3.7( \pm 3.049)$ & $p=0.836$ \\
\hline$>5$ & $8(47.1)$ & $9(52.9)$ & $17(27)$ & & \\
\hline EVA Masétero d & & & & & \\
\hline$\leq 5$ & $22(46.8)$ & 25(53.2) & $47(74.6)$ & $3.3( \pm 2.579)$ & $p=0.514$ \\
\hline$>5$ & $9(56.3)$ & $7(43.8)$ & $16(25.4)$ & & \\
\hline EVA Masétero & & & & & \\
\hline$\leq 5$ & $23(50)$ & $23(50)$ & $46(73)$ & $4.3( \pm 2.465)$ & $p=0.836$ \\
\hline$>5$ & $8(47.1)$ & $9(52.9)$ & $17(27)$ & & \\
\hline Apertura activa & olor inicial & mm) & & & \\
\hline$<40 \mathrm{~mm}$ & $11(50)$ & $11(50)$ & $22(34.9)$ & $41( \pm 7.516)$ & $p=0.926$ \\
\hline$\geq 40 \mathrm{~mm}$ & $20(48.8)$ & $21(51.2)$ & $41(65.1)$ & & \\
\hline Apertura activa & da inicial ( & & & & \\
\hline$<40 \mathrm{~mm}$ & $4(36.4)$ & $7(63.6)$ & $11(17.5)$ & $46( \pm 7.768)$ & $p=0.348$ \\
\hline$\geq 40 \mathrm{~mm}$ & $27(51.9)$ & $25(48.1)$ & $52(82.5)$ & & \\
\hline Apertura pasive & al (mm) & & & & \\
\hline$<40 \mathrm{~mm}$ & 2(33.3) & $4(66.7)$ & $6(9.5)$ & $49( \pm 7.337)$ & $p=0,414$ \\
\hline$\geq 40 \mathrm{~mm}$ & $29(50.9)$ & $28(49.1)$ & $57(90.5)$ & & \\
\hline Fuerza mastica & nicial (kg) & & & & \\
\hline$<15 \mathrm{~kg}$ & $9(45)$ & $11(55)$ & $20(32.3)$ & $20( \pm 9.478)$ & $p=0.587$ \\
\hline$\geq 15 \mathrm{~kg}$ & $22(52.4)$ & $20(47.6)$ & $42(67.7)$ & & \\
\hline
\end{tabular}

$\overline{\mathbf{E V A}}=$ escala visual análoga, $\mathbf{d e r}=$ derecha, $\mathbf{i z q}=i z q u i e r d a, \mathbf{m m}=$ milímetros, $\mathbf{k g}=$ kilógramos.

En la primera sesión, los valores iniciales para las variables dolor en ATM y maséteros derechos e izquierdos se observaron en su mayoría rangos $\leq 5$ en la escala visual análoga (EVA). Adicionalmente, para las variables iniciales de apertura activa sin dolor, apertura activa forzada y pasiva hubo una mayor cantidad de pacientes con rangos $\geq 40$ $\mathrm{mm}$. Finalmente, para la variable fuerza masticatoria inicial hubo una mayor cantidad de pacientes con rangos de $\geq 15 \mathrm{~kg}$ (Tabla 4 ).

Para la respuesta terapéutica, en el grupo TENS se encontraron diferencias significativas para dolor en la ATM derecha $(p=0.0351)$ e izquierda $(p<0.0001)$, dolor en el masétero derecho $(p=$ $0.0023)$ e izquierdo $(p=<0.0001)$, apertura activa sin dolor $(p=0.05)$ y fuerza masticatoria $(p=$ $<0.0001)$. Para el grupo CONV se encontraron diferencias significativas para dolor en la ATM iz- 

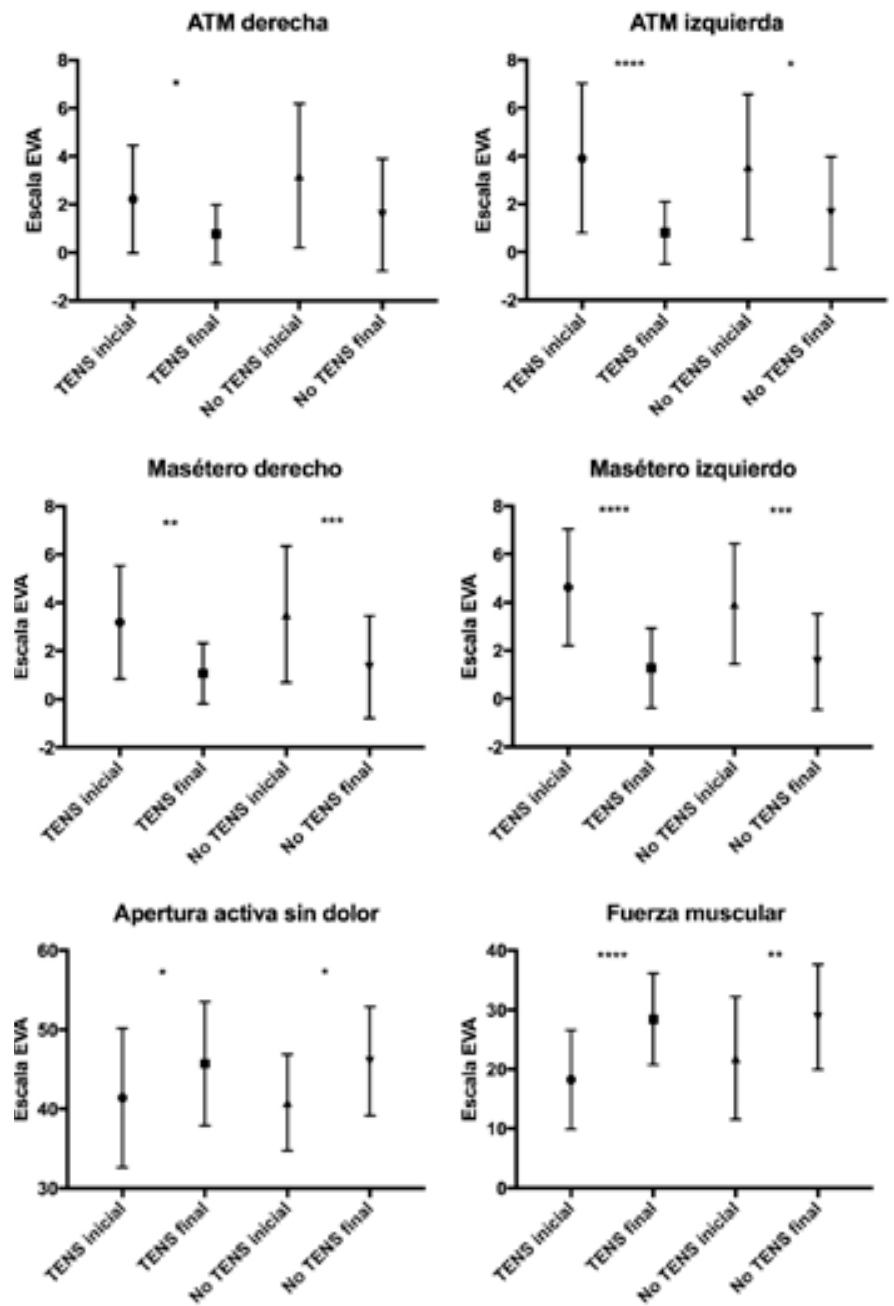

Figura 2:

$\mathbf{A}=$ Dolor ATM Derecha inicial vs final $\mathbf{B}=$ Dolor ATM Izquierda inicial vs final $\mathbf{C}=$ Dolor Masétero Derecho inicial vs final $\mathbf{D}=$ Dolor masétero Izquierdo inicial vs final $\mathbf{E}=$ Apertura activa sin dolor Inicial vs final. $\mathbf{F}=$ Fuerza masticatoria Inicial vs final. $*=$ Significativo, $* *=$ Muy significativo, $* * *=$ Altamente significativo, $* * * *=$ Extremadamente significativo.

quierda $(p=0.029)$, dolor en el masétero derecho $(p=0.0009)$ e izquierdo $(p=0.0004)$, apertura activa sin dolor $(p=0.0143)$ y fuerza masticatoria $(p=0.0056)$ (Figura 2.A-F). Adicionalmente, exceptuando el dolor en el masétero derecho, los valores $p$ en el grupo TENS fueron más significativos que en el grupo CONV.

Para las variables medidas inmediatamente luego de aplicar TENS(pre-TENS versus post-TENS), encontramos diferencias significativas para dolor en la ATM derecha $(p=<0.0001)$ e izquierda $(p=$ $<0.0001)$ iniciales, dolor en las ATM derecha ( $p$ $=0.004)$ e izquierda $(p=0.007)$ finales, dolor en los maséteros derecho $(p=0.002)$ e izquierdo $(p$
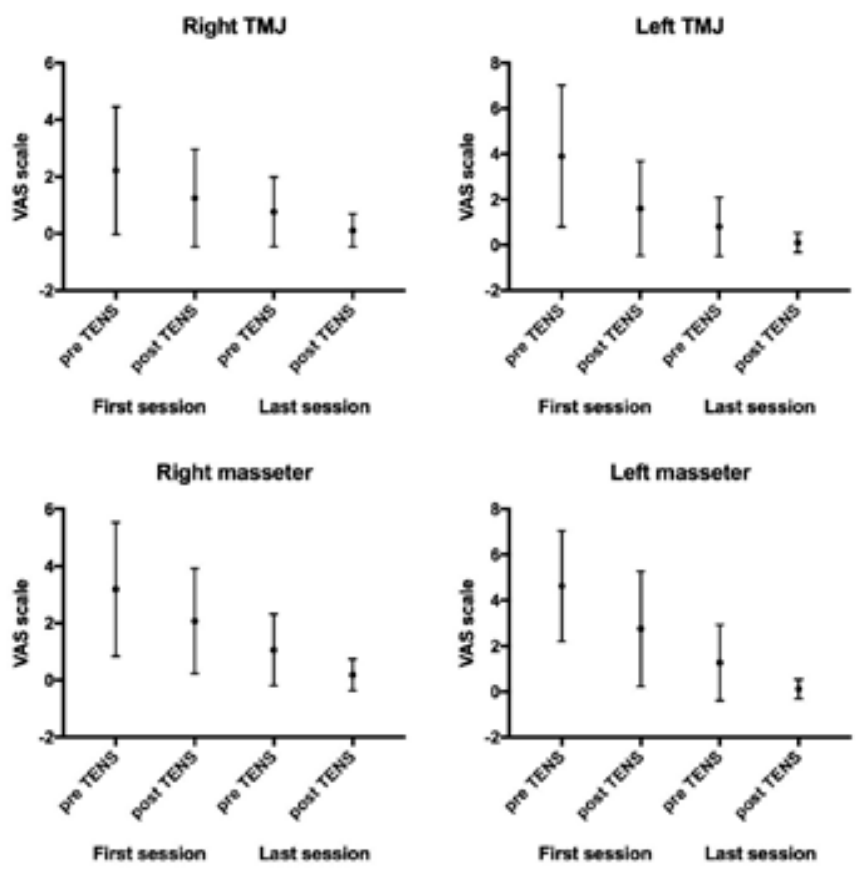

Figura 3:

$\mathbf{A}=$ Dolor ATM derecha inicial Pre-TENS vs Post-TENS y final Pre-TENS vs Post-TENS. B= Dolor ATM izquierda inicial Pre-TENS vs Post-TENS y Final Pre-TENS vs PostTENS. $\mathbf{C}=$ Dolor Masétero derecho inicial Pre-TENS vs Post-TENS y final Pre-TENS vs Post-TENS. $\mathbf{D}=$ Dolor Masétero izquierdo inicial Pre-TENS vs Post-TENS y final PreTENS vs Post-TENS.

$*=$ Significativo, $* *=$ Muy significativo, $* * *=$ Altamente significativo, $* * * *=$ Extremadamente significativo.

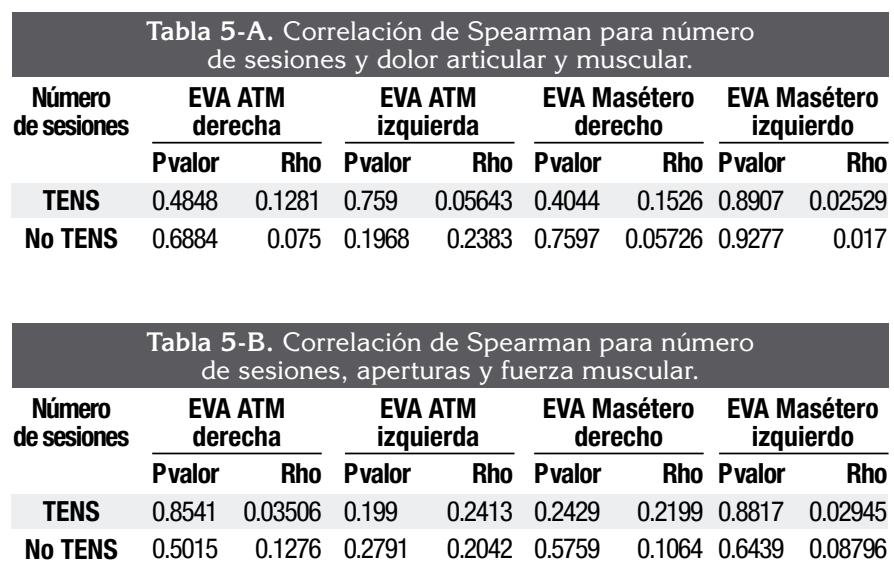

ATM=articulación temporomandibular, $\mathbf{R h o}=$ coeficiente de correlación de Spearman, TENS=transcutaneous electrical nerve stimulation.

Tabla 6. Correlación de Spearman para edad y aperturas.

\begin{tabular}{|c|c|c|c|c|c|c|}
\hline & \multicolumn{2}{|c|}{$\begin{array}{c}\text { Apertura } \\
\text { pasiva final }\end{array}$} & \multicolumn{2}{|c|}{$\begin{array}{l}\text { Apertura activa } \\
\text { sin dolor final }\end{array}$} & \multicolumn{2}{|c|}{$\begin{array}{l}\text { Apertura activa } \\
\text { forzada final }\end{array}$} \\
\hline & Pvalor & Rho & Pvalor & Rho & Pvalor & Rho \\
\hline Iad & $0.022^{*}$ & 0.296 & $0.016^{\star}$ & 0.31 & $0.01^{*}$ & 0.332 \\
\hline
\end{tabular}

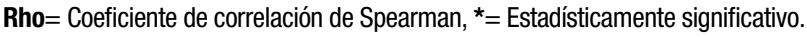


$=0.001)$ iniciales, y dolor en los maséteros derecho $(p=0.001)$ e izquierdo $(p=0.002)$ finales (Figura 3.A-D).

Al establecer correlación entre el número de sesiones y la respuesta terapéutica en ambos grupos, no se encontró correlación en relación a ninguna de las variables (Tabla 5.A-B).

Al establecer correlación entre la edad y otras variables, se encontraron diferencias estadísticamente significativas para apertura activa sin dolor final $(p=0.016)$, apertura activa forzada final $(p=0.01)$ y apertura pasiva final $(p=0.022)$ (Tabla 6), indicando que a mayor edad los sujetos presentaron menor mejora en los valores de apertura bucal.

\section{DISCUSIÓN}

El tratamiento conservador o no invasivo es la terapia de primera línea para el manejo de los TTM ${ }^{(24)}$, dentro del cual la terapia convencional, la cual incluye el uso de ARF y TO, es la más usada. Por otro lado, aunque con menor frecuencia, se ha descrito el uso de TENS como terapia complementaria ${ }^{(25)}$. En la literatura inglesa se ha utilizado frecuentemente el diseño de ensayo clínico controlado ${ }^{(14 ; 15 ; 20)}$, dentro de los cuales sólo un estudio plantea y avala al TENS como terapia complementaria ${ }^{(19)}$. En esta investigación se tuvo como objetivo determinar la respuesta terapéutica del TENS como complemento a la terapia convencional (TC/TENS) en el tratamiento de pacientes con TTM comparado con pacientes que recibieron terapia convencional (TC) exclusiva.

Los individuos del presente estudio tuvieron un promedio de edad de 29 años, con un rango entre los 14 y 69 años, similar a lo descrito en otros estudios con rangos de edad entre 18 y 60 años. El porcentaje de mujeres $(87.3 \%)$ fue mayor respecto a hombres (12.7\%), lo cual es similar a lo descrito en la literatura en la que representan generalmente más del $60 \%$ del total de la muestra ${ }^{(15 ; 19 ; 20)}$.

Los valores iniciales promedio de dolor en EVA descritos en el presente estudio fueron 3.7 para dolor articular y 4.3 para dolor muscular, los cuales son similares a los reportados en dos estudios ${ }^{(26 ; 27)}$, pero menores a los reportados en otros cuatro los cuales oscilan desde los 4.9 hasta los 8 en escala visual análoga $(19 ; 28 ; 29 ; 30)$, sin embargo, fue suficiente para ver la potencia estadística de los resultados. En cuan- to a rangos de apertura se obtuvo inicialmente un promedio de $41 \mathrm{~mm}$ para apertura activa sin dolor, $46 \mathrm{~mm}$ para apertura activa forzada y $49 \mathrm{~mm}$ para apertura pasiva, lo cual se encuentra dentro de los rangos descritos por tres estudios ${ }^{(31 ; 20 ; 32)}$, pero por sobre los descritos otros tres estudios en que se registraron valores desde los $32 \mathrm{~mm}^{(33 ; 34 ; 35)}$. El valor inicial de fuerza masticatoria fue en promedio de 20 $\mathrm{kg}$, menor que el descrito en un estudio en el cual se reportan valores promedio sobre $24 \mathrm{~kg}$ en caninos (36), pero similar al reportado en estudios en pacientes con TTM y abfracciones en que la fuerza masticatoria fue medida en primeros molares ${ }^{(37)}$.

Los principales hallazgos de este estudio fueron que en el grupo TENS se encontraron respuestas terapéuticas con diferencias estadísticamente significativas para el dolor de ATM derecha e izquierda, dolor de masétero derecho e izquierdo, apertura activa sin dolor y fuerza masticatoria, mientras que para el grupo CONV se encontraron iguales respuestas terapéuticas que en el anterior, a excepción del dolor en ATM derecha. Adicionalmente, en el grupo TENS se encontraron respuestas estadísticamente significativas para las variables dolor en ATM y maséteros inmediatamente luego de la aplicación del TENS. Estos resultados se explican ya que por un lado se ha reportado que el tratamiento conservador por sí solo logra sanar al paciente ${ }^{(25 ; 28 ; 38 ; 39,33)}$, y por otro que se les atribuye a los TTM un carácter autolimitante por lo que varios pacientes deberían naturalmente mejorar en el largo plazo incluso si no se les aplica terapia alguna, lo cual podría además evidenciar la existencia del efecto placebo que idealmente debe pesquisarse ${ }^{(31)}$.

Adicionalmente el TENS es una buena alternativa de terapia complementaria, con un mayor efecto en el alivio del dolor en el corto que en el largo plazo, así como ocurrió en este estudio al comparar mediciones previas e inmediatamente posteriores a la aplicación de TENS para las variables de dolor en ATM y maséteros $(14 ; 15 ; 25 ; 40)$. Este efecto inmediato en el corto plazo se ha descrito como facilitador en la manipulación de la mandíbula del paciente en caso ser necesario realizar registros para un tratamiento basado en TO como el realizado dentro del tratamiento convencional en este estudio ${ }^{(12)}$.

En ambos grupos estudiados no hubo valores estadísticamente significativos en la respuesta terapéutica para las variables apertura activa forzada ni apertura pasiva. Ello puede deberse a que por un lado la apertura forzada no es funcional y por otro la apertura pa- 
siva depende del operador, además éstas no tienen relación con el tipo de TTM ni la severidad del mismo ${ }^{(41)}$.

No se encontraron correlaciones estadísticamente significativas entre el número de sesiones y la respuesta terapéutica de las variables medidas en ambos grupos. Lo cual se debe en primera instancia a que el protocolo usado fue el mismo para ambos grupos no generando diferencias en el número de sesiones de cada uno, y en segunda instancia a que tanto la terapia convencional con TO y ARF al igual que la complementada con TENS poseen tiempos necesarios similares para lograr una respuesta terapéutica significativa ${ }^{(39)}$. En este estudio el tratamiento duró 4 semanas y el seguimiento se realizó por 8 semanas duración similar a lo reportado en la literatura a la fecha ${ }^{(40)}$. Es importante comentar que además hay una falta de protocolos universales para la aplicación de TENS en estudios clínicos ${ }^{(9)}$.

La edad demostró correlaciones estadísticamente significativas con la respuesta terapéutica de las variables apertura activa sin dolor, activa forzada y pasiva. Esto indica que a mayor edad hay menor rango de mejora de éstas variables, coincidiendo con la literatura previa ${ }^{(42)}$. Estos resultados pueden explicarse debido a que se ha descrito que pacientes jóvenes responden mejor al TENS que los adultos mayores (26). Más allá, al aumentar la edad, los músculos se debilitan, disminuyendo la fuerza con la que se realiza la apertura ${ }^{(43)}$ y los tejidos conectivos, incluyendo el cartílago, se van poniendo más rígidos adquiriendo menores rangos de movimiento ${ }^{(44)}$.

Por otro lado la etiología de los TTM tiene una evidente relación con las características psicosociales del paciente ${ }^{(45)}$, por lo que pudieron haber ocurrido eventos importantes que alteraron a algunos pacientes, los cuales modificaron el resultado final del tratamiento o hicieron que en algunos casos se hubiera requerido otro tipo de tratamiento según las necesidades de cada paciente. Cabe destacar que la aplicación de TENS en otras condiciones orofaciales ha sido asociada con una mayor adhesión al tratamiento (25). Considerando estos reportes, su bajo costo, la seguridad de su uso y su nula interacción con fármacos, es que el TENS ha sido recomendado como un buen elemento complementario para el manejo del dolor de la población ${ }^{(46)}$.

Dentro de las fortalezas del presente estudio encontramos el gran número de variables medidas, incluso ningún estudio previo en TENS había medido fuerza masticatoria ${ }^{(40)}$; la cantidad de pacientes analizados, ya que solo un estudio lo supera por 30 pacientes, y lo iguala en el seguimiento de 60 días ${ }^{(20)}$. Sin embargo, también presenta limitaciones, como el haber realizado un censo de la escuela de Odontología y no incluir a consultorios, hospitales u otros centros de salud; la no asistencia a todos los controles por parte de los pacientes en el tiempo que debían acudir, alterando así el seguimiento; la falta de adhesión a las indicaciones del tratamiento y el hecho de que hubo pacientes que empeoraron en cuanto al dolor registrado en la primera sesión. Considerando lo anterior los resultados de este estudio son aplicables solo a los pacientes que acuden a la Escuela de Odontología de la Universidad de Valparaíso, ya que los controles vienen de la misma población y no es posible extrapolarlos a la población general.

Para futuros estudios se sugiere realizar un diseño prospectivo ensayo clínico controlado aleatorizado, además incluir un grupo placebo, en el cual se utilice un TENS placebo, para determinar y cuantificar si éste afecta la respuesta a la terapia. Finalmente para evaluar el estado psicosocial del paciente durante el tratamiento, mediante un instrumento validado con el fin de poder determinar si logra modificar y alterar los resultados obtenidos, además de evaluar el efecto inmediato del TENS en la ansiedad del paciente, y si éste mejora la adhesión del paciente al tratamiento.

\section{En términos específicos:}

1. Se obtuvieron mejores respuestas terapéuticas en pacientes jóvenes al evaluar apertura mandibular activa sin dolor, activa forzada y pasiva.

2. No se encontraron diferencias en las respuestas terapéuticas reportadas en ambos grupos, a excepción del dolor articular en la ATM derecha en el grupo convencional, el cual no tuvo una respuesta terapéutica significativa. Adicionalmente no se obtuvieron respuestas terapéuticas significativas al medir la apertura activa forzada y apertura pasiva.

3. Finalmente, dentro de la sesión, se observó una respuesta terapéutica positiva al evaluar dolor articular y muscular inmediatamente después de la aplicación de TENS.

\section{En términos generales:}

Se encontraron respuestas terapéuticas similares en 
pacientes tratados con terapia convencional exclusiva así como complementada con TENS. Esto nos permite concluir que el uso de TENS no modifica la respuesta terapéutica a largo plazo. Sin embargo, la aplicación del TENS produce una reducción inmediata que ayuda al manejo del dolor en el corto plazo.

\section{AGRADECIMIENTOS}

Nos gustaría agradecer a Luis Guerrero, quien participó en la evaluación y tratamiento de los pacientes. También a Fernando Weiss (Q.E.P.D.), quien ayudó en la recolección de la información. Este estudio utilizó la base de datos de la Clínica de Especialidades de la Escuela de Odontología de la Universidad de Valparaíso.

\section{REFERENCIAS BIBLIOGRÁFICAS}

1. De Leeuw R, Klasser GD. Orofacial Pain: Guidelines for Assessment, Diagnosis, and Management. Quintessense: Chicago. Sixth Edition; 2018.

2. Schiffman E, Ohrbach R, Truelove E, Look J, Anderson G, Goulet JP, et al. Diagnostic Criteria for Temporomandibular Disorders (DC/TMD) for Clinical and Research Applications: recommendations of the International RDC/TMD Consortium Network* and Orofacial Pain Special Interest Group. J Oral Facial Pain Headache 2014; 28(1):6-27.

3. Chipaila N, Sgolastra F, Spadaro A, Pietropaoli D, Masci $C$, Cattaneo R, et al . The effects of ULF-TENS stimulation on gnathology: The state of the art. Cranio - J Craniomandib Pract 2014; 32(2):118-30.

4. Chisnoiu AM, Picos AM, Popa S, Chisnoiu PD, Lascu L, Picos A., et al. Factors involved in the etiology of temporomandibular disorders - a literature review. Clujul Med 2015; 88(4):473-78.

5. Dubner R, Ohrbach R, Dworkin SF. The Evolution of TMD Diagnosis: : Past, Present, Future. J Dent Res 2016; 95(10):1093-101.

6. Politti F, Casellato C, Kalytczak MM, Garcia MBS, Biasotto-Gonzalez DA. Characteristics of EMG frequency bands in temporomandibullar disoders patients. J Electromyogr Kinesiol 2016; 31:119-25.

7. Wieckiewicz M, Boening K, Wiland P, Shiau YY, Paradowska-Stolarz A. Reported concepts for the treatment modalities and pain management of temporomandibular disorders. J Headache Pain 2015; 16:106-18.

8. Carlson CR, Bertrand PM, Ehrlich D, Maxwell W, Burton RG. Physical self-regulation training for the management of temporomandibular disorders. J Orofac Pain 2001; 15(1):47-55.

9. Ferreira APL, Costa DRA, Oliveira AIS, Carvalho EAN, Conti PCR, Costa YM, et al. Short-term transcutaneous electrical nerve stimulation reduces pain and improves the masticatory muscle activity in temporomandibular disorder patients: a randomized controlled trial. J Appl Oral Sci 2017; 25(2):112-20.
10. Vance G, Dailey D, Rakel B, Sluka K. Using TENS for pain control: the state of the evidence. Pain management 2014; 4(3): 197-209.

11. Coutaux, A. Non-pharmacological treatments for pain relief: TENS and acupuncture. Joint Bone Spine 2017; 84(6): 657-61.

12. Esclassan R, Rumerio A, Monsarrat P, Combadazou J, Champion J, Destruhaut $F$, et al. Optimal duration of ultra low frequency-transcutaneous electrical nerve stimulation (ULF-TENS) therapy for muscular relaxation in neuromuscular occlusion: A preliminary clinical study. CRANIO® 2016; 35(3): 175-79.

13. Mummolo S, Nota A, Tecco S, Caruso S, Marchetti E, Marzo G, et al. Ultra-low-frequency transcutaneous electric nerve stimulation (ULF-TENS) in subjects with craniofacial pain: A retrospective study. CRANIO®; 2018; Published online 8 october of 2018: 1-6.

14. Rodrigues D, Siriani AO, Bérzin F. Effect of conventional TENS on pain and electromyographic activity of masticatory muscles in TMD patients. Braz Oral Res 2004; 18(4):290-95.

15. Núñez SC, Garcez AS, Suzuki SS, Ribeiro MS. Management of Mouth Opening in Patients with Temporomandibular Disorders through Low-Level Laser Therapy and Transcutaneous Electrical Neural Stimulation. Photomed Laser Surg 2006; 24(1):45-9.

16. Butts R, Dunning J, Pavkovich R, Mettille J, Mourad F. Conservative management of temporomandibular dysfunction: A literature review with implications for clinical practice guidelines (Narrative review part 2). Vol. 21, Journal of Bodywork and Movement Therapies 2017; pp. 541-48.

17. Awan KH, Patil S. The role of transcutaneous electrical nerve stimulation in the management of temporomandibular joint disorder. J Contemp Dent Pract 2015; 16(10): 984-86.

18. De Giorgi I, Castroflorio T, Sartoris B, Deregibus A. The use of conventional transcutaneous electrical nerve stimulation in chronic facial myalgia patients. Clinical Oral Investigations 2016; 21(1): 275-80.

19. Shanavas M, Chatra L, Shenai P, Rao PK, Jagathish V, Kumar SP, et al. Transcutaneous electrical nerve stimulation therapy: An adjuvant pain controlling modality in TMD patients - A clinical study. Dent Res J (Isfahan) 2014; $11(6): 676-79$.

20. Rai S, Ranjan V, Misra D, Panjwani S. Management of myofascial pain by therapeutic ultrasound and transcutaneous electrical nerve stimulation: A comparative study. Eur J Dent 2016; 10(1):46-53.

21. Rezazadeh F, Hajian K, Shahidi S, Piroozi S. Comparison of the Effects of Transcutaneous Electrical Nerve Stimulation and Low-Level Laser Therapy on Drug-Resistant Temporomandibular Disorders. Journal of Dentistry 2017; 18(3): 187-92.

22. Dworkin SF, LeResche L. Research diagnostic criteria for temporomandibular disorders: review, criteria, examinations and specifications, critique. J Craniomandib Disord 1992; 6(4):301-55.

23. Tong KC, Lo SK, Cheing GL. Alternating Frequencies of Transcutaneous Electric Nerve Stimulation: Does it Produce Greater Analgesic Effects on Mechanical and Thermal Pain Thresholds? Arch Phys Med Rehabil 
W. Meeder Bella, C. León-Morales, O. Leissner, E. Vergara-Bruna, M. Maulén-Yañez, W. A. González-Arriagada Estimulación nerviosa eléctrica transcutánea como complemento a la terapia convencional en pacientes con trastornos temporomandibulares: un estudio caso-control

2007; 88(10):1344-49.

24. Abouelhuda A, Khalifa A, Kim Y, Hegazy S. Non-invasive different modalities of treatment for temporomandibular disorders: review of literature. J Korean Assoc Oral Maxillofac Surg 2018; 44(2):43-51.

25. Kasat V, Gupta A, Ladda R, Kathariya M, Saluja H, Farooqui AA. Transcutaneous electric nerve stimulation (TENS) in dentistry- A review. J Clin Exp Dent 2014; 6(5):562-68.

26. Bergeron-Vézina K, Corriveau H, Martel M, Harvey MP, Léonard G. High- and low-frequency transcutaneous electrical nerve stimulation does not reduce experimental pain in elderly individuals. Pain 2015; 156(10):2093-9.

27. Madani A, Ahrari F, Fallahrastegar A, Daghestani N. A randomized clinical trial comparing the efficacy of low-level laser therapy (LLLT) and laser acupuncture therapy (LAT) in patients with temporomandibular disorders. Lasers in Medical Science 2019; Online Aug 8. doi: $10.1007 / \mathrm{s} 10103-019-02837-\mathrm{x}$.

28. Van Grootel RJ, Buchner R, Wismeijer D, Van Der Glas HW. Towards an optimal therapy strategy for myogenous TMD, physiotherapy compared with occlusal splint therapy in an $\mathrm{RCT}$ with therapy-and-patient-specific treatment durations. BMC Musculoskelet Disord 2017; 18: 76-93.

29. Khairnar S, Bhate K, Kshirsagar K, Jagtap B, Kakodkar P. Comparative evaluation of low-level laser therapy and ultrasound heat therapy in reducing temporomandibular joint disorder pain. J Dent Anesth Pain Med 2019; 19(5):289-94.

30. Valentino R, Cioffi I, Vollaro S, Cimino R, Baiano R, Michelotti A. Jaw muscle activity patterns in women with chronic TMD myalgia during standardized clenching and chewing tasks. CRANIO® 2019; 21:1-7.

31. Kato MT, Kogawa EM, Santos CN, Conti PC. TENS and low-level laser therapy in the management of temporomandibular disorders. J Appl Oral Sci 2006; 14(2):130-35.

32. Thorp JN, Willson J. Thoracic spine manipulation did not improve maximal mouth opening in participants with temporomandibular dysfunction. Physiotherapy Research International. 2019; 15:e1824.

33. Patil S, Iyengar AR, Kotni RM, Joshi RK. Evaluation of Efficacy of Ultrasonography in the Assessment of Transcutaneous Electrical Nerve Stimulation in Subjects with Myositis and Myofascial Pain. Korean J Pain 2016; 29(1):12-17.

34. Vrbanovi囚 E, Alajbeg IZ. Long-term Effectiveness of Occlusal Splint Therapy Compared to Placebo in Patients with Chronic Temporomandibular Disorders. Acta Stomatol Croat 2019; 53(3):195-206.

35. Abbasgholizadeh ZS, Evren B, Ozkan Y. Evaluation of the efficacy of different treatment modalities for painful temporomandibular disorders. International Journal of Oral and Maxillofacial Surgery 2019; Sep 20. pii: S09015027(19)31295-0.

36. Curiqueo A, Salamanca C, Borie E, Navarro P. Evaluación de la Fuerza Masticatoria Máxima Funcional en Adultos Jóvenes Chilenos. Int J Odontostomatol 2015. 9(3):443-47.

37. Rodrigues AMB, Magri LV, Moreira MA, de Sousa MD. Are the bite force and electromyographic activity altered in muscle TMD patients with abfraction lesions?. J
Craniomandib Disord 2017; DOI:10.1080/08869634. 2017.1407116.

38. Romero-Reyes M, Uyanik JM. Orofacial pain management: Current perspectives. Vol. 7, Journal of Pain Research 2014; pp. 99-115.

39. Shaffer SM, Brismée JM, Sizer PS, Courtney CA. Temporomandibular disorders. Part 2: conservative management. J Man Manip Ther 2014; 22(1):13-23.

40. Fertout A, Manière-Ezvan A, Lupi L, Ehrmann E. Management of temporomandibular disorders with transcutaneous electrical nerve stimulation: A systematic review. CRANIO® 2019; Nov 9:1-12.

41. Gomes CAF, Dibai-Filho AV, Da Silva JR, De Oliveira PM, Politti F, Biasotto-Gonzalez DA. Correlation between severity of temporomandibular disorder and mandibular range of motion. J Bodyw Mov Ther 2014; 18(2):306-310.

42. Agrawal J, Shenai PK, Chatra L, Kumar PY. Evaluation of normal range of mouth opening using three finger index: South India perspective study. Indian J Dent Res 2015; 26(4):361-65.

43. Wakasugi Y, Tohara H, Machida N, Nakane A, Minakuchi $\mathrm{S}$. Can grip strength and/or walking speed be simple indicators of the deterioration in tongue pressure and jaw opening force in older individuals? Gerodontology 2017; 34(4):455-59.

44. Roberts S, Colombier P, Sowman A, Mennan C, Rölfing JHD, Guicheux J, et al. Ageing in the musculoskeletal system: Cellular function and dysfunction throughout life. Acta Orthop 2016; 87:15-25.

45. Miettinen O, Lahti S, Sipilä K. Psychosocial aspects of temporomandibular disorders and oral health-related quality-of-life. Acta Odontol Scand 2012; 70(4):331-36.

46. Tashani O, Johnson MI. Transcutaneous electrical nerve stimulation (TENS) a possible aid for pain relief in developing countries?. Libyan Journal of Medicine 2009; (4) pp. 62-65

\section{AUTOR DE CORRESPONDENCIA:}

Walther Meeder-Bella, DDS, MSc, Departamento de Fisiología Oral y Trastornos Temporomandibulares, Facultad de Odontología, Universidad de Valparaíso, Subida Leopoldo Carvallo \#211, Playa Ancha, Valparaíso, Chile. +56 322508531. walther.meeder@uv.cl.

\section{CONFLICTOS DE INTERÉS}

Los autores declaran que no hay conflicto de intereses.

\section{APROBACIÓN ÉTICA}

Estudio aprobado por parte del Comité de Bioética de la Facultad de Odontología de la Universidad de Valparaíso (código: PostG-08-17) y llevado a cabo de acuerdo a la Declaración de Helsinki. Los pacientes involucrados dieron su consentimiento informado escrito para participar, en caso de niños, éste fue dado por sus padres. 